University of Wollongong

Research Online

Faculty of Engineering and Information

Faculty of Engineering and Information

Sciences - Papers: Part B

Sciences

2018

Effects of orthopedic insoles on static balance of older adults wearing thick socks

Christina Ma

The Hong Kong Polytechnic University

Duo Wong

The Hong Kong Polytechnic University

Anson Wan

The Hong Kong Polytechnic University

Winson Lee

University of Wollongong, ccwlee@uow.edu.au

Follow this and additional works at: https://ro.uow.edu.au/eispapers1

Part of the Engineering Commons, and the Science and Technology Studies Commons

Research Online is the open access institutional repository for the University of Wollongong. For further information contact the UOW Library: research-pubs@uow.edu.au 


\title{
Effects of orthopedic insoles on static balance of older adults wearing thick socks
}

\author{
Abstract \\ Background: The wearing of socks and insoles may affect the ability of the foot to detect tactile input \\ influencing postural balance. Objectives: The aim of this study was to investigate whether (1) thick socks \\ adversely affected the elderly postural balance and (2) orthopedic insoles could improve the elderly \\ postural balance while wearing thick socks. Study design: Repeated-measures study design. Methods: In \\ total, 14 healthy older adults were recruited. A monofilament test was conducted to evaluate foot plantar \\ sensation with and without thick socks. Subjects then performed the Romberg tests under three \\ conditions: (1) barefoot, (2) with socks only, and (3) with both socks and insoles. Postural balance was \\ assessed by measuring the center of pressure movement during standing in each experimental condition. \\ Results: Thick socks significantly decreased the monofilament score $(p<0.001)$, suggesting reduction in \\ ability to detect external forces. All center of pressure parameters increased significantly while wearing \\ thick socks $(p<0.017)$, implying reduction of postural stability. They then decreased significantly with the \\ additional use of insoles $(p<0.017)$. Conclusion: Previous studies have documented the changes in \\ plantar pressure distribution with the use of orthopedic insoles. This study further suggests that such \\ changes in contact mechanics could produce some balance-improving effects, which appears not to have \\ been reported earlier. Clinical relevance: Wearing thick socks reduces plantar pressure sensitivity and \\ increases postural sway which may increase risk of falls. Orthopedic insoles and footwear with similar \\ design could potentially be a cost-effective method in maintaining postural balance when wearing thick \\ socks.

\section{Disciplines} \\ Engineering | Science and Technology Studies

\section{Publication Details} \\ Ma, C. Zong-Hao., Wong, D. Wai-Chi., Wan, A. Hong-Ping. \& Lee, W. Chiu-Chun . (2018). Effects of \\ orthopedic insoles on static balance of older adults wearing thick socks. Prosthetics and Orthotics \\ International, 42 (3), 357-362.
}




\section{Effects of Orthopaedic Insoles on Static Balance of Older}

\section{Adults Wearing Thick Socks}

\section{Abstract}

Study Design: Repeated measures study design.

Background: The wearing of socks and insoles may affect the ability of the foot to detect tactile input influencing postural balance.

Objectives: The aim of this study was to investigate if 1) thick socks adversely affected the elderly postural balance, and 2) orthopaedic insoles could improve the elderly postural balance while wearing thick socks.

Methods: Fourteen healthy older adults were recruited. A monofilament test was conducted to evaluate foot plantar sensation with and without thick socks. Subjects then performed the Romberg tests under 3 conditions: (1) barefoot; (2) with socks only; (3) with both socks and insoles. Postural balance was assessed by measuring the center of pressure (COP) movement during standing in each experimental condition.

Results: Thick socks significantly decreased the monofilament score $(p<0.001)$, suggesting reduction in ability to detect external forces. All COP parameters increased significantly while wearing thick socks $(\mathrm{p}<0.017)$, implying reduction of postural stability. They then decreased significantly with the additional use of insoles $(p<0.017)$.

Conclusions: Previous studies have documented the changes in plantar pressure distribution with the use of orthopaedic insoles. This study further suggests such changes 
in contact mechanics could produce some balance-improving effects, which appears not have been reported earlier.

Word count: 199 words.

\section{Clinical Relevance:}

Orthopaedic insoles were traditionally used to treat foot pain/deformity. This study showed they could also reduce static body sway of older adults with thick socks. Increased postural sway has been suggested to be linked to risk of falls. Orthopaedic insoles and footwear with similar design could potentially be a cost-effective method in improving postural balance.

Word count: 50 words.

Keywords: balance, center of pressure, elderly, fall, orthopaedic insoles, postural stability

\section{Background}

Falls and fall-induced injuries are associated with high morbidity and mortality rate, significant reduction of quality of life, and enormous hospitalization expense. ${ }^{1}$ Plantar tactile sensation plays a critical role in balance control, as it provides the central nervous systems with instantaneous and continuous information on the characteristics of the ground support surface and the movement of the body relative to the foot. ${ }^{2}$ Declined plantar tactile sensation can induce poor balance and predispose risk of falls. ${ }^{2}$ Aging, diabetic neuropathy, and rheumatoid arthritis can lead to impairment in plantar tactile 
sensation. ${ }^{3}$ In addition, some footwear could reduce the tactile input to the plantar surface of foot and consequently influence the postural stability. ${ }^{4}$

Wearing thick or even multiple layers of socks could improve comfort ${ }^{4}$ and protect the feet from frostbite during cold weather. ${ }^{5}$ However, the use of socks could be linked to increased falling risks. ${ }^{4,6}$ The reason behind this phenomenon could be that the soft materials under the feet could attenuate the plantar tactile sensory input, ${ }^{4,6}$ which is crucial for balance control. This mechanism could also explain the poorer postural stability caused by either wearing shoes with soft soles ${ }^{4}$ or standing on a soft foam surface. $^{7}$

Previous studies have attempted to modify the physical properties of the footwear in order to aid balance, as reviewed by Hatton et al. ${ }^{8}$ Shoe soles and insoles made of hard materials were shown to have positive effects on improving balance. ${ }^{8}$ It was also reported that adhering a flexible plastic tubing to the margin of foot sole ${ }^{9}$ and some small semirigid plastic nubs or spikes to the insoles ${ }^{10}$ enhanced plantar sensory input and balance. However, these designs tended to cause some pain and discomfort and altered the normal gait patterns of the users, ${ }^{10}$ which limited their application in daily life. Meanwhile, some other studies suggested that vibrating insoles can enhance plantar sensory input and thus improve balance. ${ }^{11}$ As the vibrator was made of rigid steel, however, users complained of pain and discomfort when using vibrating insoles. ${ }^{8}$ It has also been suggested that the economic and practicality problems associated with vibrating insoles may outweigh their beneficial effects on balance control. ${ }^{8}$

Orthopaedic insoles are conventionally used to treat foot pain and correct foot deformity in patients. ${ }^{12}$ Arch supports, metatarsal pads, and heel cups are key components of a pair 
of orthopaedic insoles. Arch supports relieve plantar fasciitis by supporting the longitudinal arch and relieving soft tissue stretch. ${ }^{13}$ Metatarsal pads relieve pain over the metatarsal heads by redistributing loadings to the metatarsal shafts. ${ }^{14}$ Heel cups help to grasp the heel in a more neutral position. ${ }^{15}$ Some studies reported that balance could be improved by reducing foot pain, ${ }^{16}$ correcting foot deformities, ${ }^{17,} 18$ and putting the foot and ankle joint in a more stable position ${ }^{15,19}$ upon using orthopaedic insoles. However, it is not known if these balance improvement effects can still be retained in people without any foot pain or deformity.

Orthopaedic insoles may also have some positive effects on balance for those without foot pain or deformity. Previous studies measuring plantar pressure distribution indicated that orthopaedic insoles increased the contact area between the foot and the support surface. $^{15,} 17$ In addition, orthopaedic insoles redistributed the plantar pressure by increasing the pressure over the metatarsal shafts and reducing the pressure over the heel and the metatarsal heads which are the commonly painful sites. ${ }^{20}$ It just happens that the midfoot metatarsal shaft region was shown to have higher tactile sensitivity than the heel and the metatarsal heads. ${ }^{3}$ The increased contact area and the elevated pressure over some more sensitive regions could enhance plantar tactile input, which gives traditional orthopaedic insoles the potential to improve balance of people with deficits of plantar tactile sensation. So far, studies have not confirmed about this hypothesis using scientific methods.

Different balance assessment tests have been applied in clinical practice, including the Brunel Balance Assessment, Berg Balance Scale, and Weight Shifting Test. ${ }^{21,22}$ In laboratory setting, the use of force plates allows assessment of balance to be more 
objective and sensitive to changes, as compare to clinical balance assessment tests. ${ }^{21}$ Increase in center of pressure (COP) movements during standing is typically considered to be an indication of decreased postural stability. ${ }^{23}$ More importantly, fallers tend to have more static postural sway than non-fallers. ${ }^{24}$

This study evaluated the effects of wearing a pair of thick socks on postural balance of older adults, then further investigated if orthopaedic insoles could produce any significant changes in postural balance while wearing thick socks. Postural balance was assessed by measuring the movement of COP. It was hypothesized that conventional orthopaedic insoles could improve postural balance of older adults, which was adversely affected by the use of thick socks. This study potentially uncovers the balance improving effects of traditional orthopaedic insoles and sheds new light on the application of a low-cost and practical solution for improving balance.

\section{Methods}

\section{Subjects}

Convenience sampling approach was used to recruit fourteen healthy older adults aged over 65 years in this study. The subjects were fully independent, living in a communitybased setting, and capable of unassisted ambulation. Subjects did not have pes planus (flat foot), pes cavus (high arch), or foot pain, as assessed by a certified orthotist. In addition, the subjects did not have self-reported history of foot injury, neurological or vestibular disorders. All subjects were able to follow the instructions and procedures of the research protocol. 
All subjects provided written informed consent before participating in the study. Ethical approval was granted from the authority of local university.

\section{Design of Insoles}

Figure 1 shows the insoles used in this study. They were commercially available (Foot Specialist Footcare \& Products Co. Ltd, Hong Kong, China) full-length insoles made of medium firm (30-35 Shore A Hardness) ethylene-vinyl acetate (EVA), with medial longitudinal arch supports and heel cups. They were originally indicated for conditions of flat foot, plantar fasciitis and metatarsalgia. A metatarsal pad was added to the prefabricated insoles just proximal to the $2^{\text {nd }}$ and $3^{\text {rd }}$ metatarsal heads. Three different heights of metatarsal pads (4, 6 and $8 \mathrm{~mm}$ ) were used. The thickest possible metatarsal pad without causing discomfort was selected for each subject. The lengths of the insoles were selected and the heights of the pads were adjusted by a certified orthotist prior to the experiment.

\section{The Socks}

A pair of thick socks, which were below the ankle joint, were used. The socks (88515016, Baleno Co. Ltd, Hong Kong, China) were commercially available, with a thickness of 4.5mm at bottom, and composed of $80.6 \%$ cotton, $16.7 \%$ polyamide and $2.7 \%$ spandex.

\section{Procedure}

This study was conducted in a university human locomotion laboratory. A Romberg test was conducted to assess the static balance following the standard procedures, ${ }^{25}$ which were shown in a previous study to have high test-retest reliability. ${ }^{26}$ The subjects were 
instructed to stand quietly on a force platform without shoes, with arms crossed resting on the opposite shoulders and eyes-closed. Subjects were instructed to stand in the center of the force platform with foot position been marked, to ensure the consistent positioning of foot over three experimental conditions. The insoles were fixed on the force platform by adhesive tapes such that the subjects stood with medial sides of the feet touching each other, following the protocols of Romberg test. ${ }^{25}$ Balance was assessed under three test conditions: 1) without socks or insoles (Condition 1), 2) with socks but without insoles (Condition 2), and 3) with both socks and insoles (Condition 3). In each testing trial, subjects were instructed to stand as still as possible for 90s. Each test condition was repeated 3 times consecutively for each subject. Between each condition, each subject was given a 10-minute rest to eliminate possible effects of fatigue. The three test conditions were coded and the testing order of the three different conditions was randomly generated for each subject.

\section{Outcome Measures}

A 5.07/10-g Semmes-Weinstein monofilament (Connecticut Bio-instruments Inc. Bronx, NY, USA) was used to assess the effects of wearing thick socks on the ability of plantar surface of the foot to detect external forces, following the testing procedures as specified in Slater et al. ${ }^{27}$ During the test, three sites at the plantar surface of foot were assessed including the hallux, the first and fifth metatarsal heads, with and without wearing the thick socks. At each site, the monofilament was pressed to the skin at 90 degrees with a force of $10 \mathrm{~g}(0.1 \mathrm{~N})$ to produce bowing for at least $1 \mathrm{~s}$, which guaranteed the applied forces be kept consistent over the experiment. Two applications and a sham application were performed at each site. The sequence of the actual and sham applications was 
randomized. The subjects were instructed to respond "yes/no" after each application to indicate if they could sense the touch of the monofilament with their eyes closed. Scores of each subject were marked from 0 to 3 based on the number of correct answers at each test site. ${ }^{27}$ The higher scores of the monofilament represented the better detection of plantar pressure stimulations. All monofilament tests were performed by the same examiner.

A force platform (OR6, Advanced Mechanical Technology, Inc., Watertown, MA, USA) sampling at $1000 \mathrm{~Hz}$ was used to measure the relative location of the COP signals to the coordinate origin of force platform. The real-time COP was recorded and used to calculate COP-based parameters. Based on the computed changes of locations of COP and their time of occurrence, the (1) mean velocity (mm/s), (2) root mean square distance (mm), (3) the 95\% confidence circle area $\left(\mathrm{mm}^{2}\right)$, (4) the 95\% confidence ellipse area $\left(\mathrm{mm}^{2}\right)$, (5) sway area $\left(\mathrm{mm}^{2}\right)$, and (6) range of COP in anteroposterior (AP) and mediolateral (ML) directions (mm) were calculated using Microsoft Excel..$^{28}$

\section{Statistical Methods}

Data analysis was performed using Statistical Package for Social Sciences (SPSS, version 25.0, IBM Corporation, Armonk, NY, USA). Wilcoxon Signed-Ranks Test was used to compare the monofilament score with and without wearing the thick socks. One-way repeated measures ANOVA with Bonferroni corrections was performed to study if significant differences existed in all measured COP parameters among the three conditions in all 14 subjects. If significant difference was found in ANOVA, post hoc pair-wise comparisons were performed by conducting t-tests with Bonferroni corrections. 
The level of significance was set at 0.05 . During data analysis, the person who analyzed data did not know the content of each test condition as conditions were coded.

\section{Results}

Fourteen elderly subjects (four females and ten males, aged 70.2 \pm 3.4 years, height $162.8 \pm 7.9 \mathrm{~cm}$, and weight $63.6 \pm 10.0 \mathrm{~kg}$ ) participated in the study.

Table 1 shows that the average monofilament score decreases significantly from 2.8 to 1.1 after using the socks $(\mathrm{p}<0.001)$. The percentage decreases of monofilament score at hallux, $1^{\text {st }}$ metatarsal head, and $5^{\text {th }}$ metatarsal head were $61.9 \%(\mathrm{p}<0.001), 63.4 \%$ $(\mathrm{p}<0.001)$, and $61.1 \%(\mathrm{p}<0.001)$, respectively.

A typical example of the COP trajectory in three experimental conditions in an elderly subject is shown in Figure 2. As shown in Figure 2A and 2B, condition 2 (with socks) induced a larger COP sway area than condition 1 (without socks). The sway area of COP was then reduced in condition 3 (using insoles, with socks continued to be put on) (Figure 2C).

Table 2 presents the quantitative data of COP parameters in 3 experimental conditions $(n=14)$. The results of one-way repeated measures ANOVA revealed the existence of significant difference in COP parameters among three experimental conditions. All COP parameters increased significantly when wearing socks (Condition 2 vs $1, \mathrm{p}<0.001$ ), then decreased significantly when wearing both socks and insoles (Condition 3 vs 2, p<0.001). No significant difference was found comparing condition 3 with condition 1 (Condition 3 vs 1$)$. 


\section{Discussion}

Falls and fall-induced injuries are major public health problems, and continuous efforts have been made to improve older population and patients' balance. Previous studies showed that orthopedic insoles increased the plantar pressure at relatively more sensitive areas over the metatarsal shafts ${ }^{20}$ and the total contact area between the foot and the footwear. ${ }^{15,17,20}$ This study further suggested that such changes in contact mechanics could lead to improved static balance of people, whose balance had been affected by the use of thick socks.

The monofilament scores indicated that the thick socks lowered the ability of the foot to sense the external forces. The bowing of the monofilament during the test ensured that the magnitude of force (i.e. $10 \mathrm{~g} / 0.1 \mathrm{~N}$ ) applied kept constant in both conditions of with and without wearing thick socks. ${ }^{29}$ The lower monofilament scores with the use of thick socks suggested that the deformation of socks may distribute the forces and reduce the mechanical pressure acting against the foot, which restricted the plantar foot to detect less mechanical stimulations at the interface between foot and ground. The increase in postural sway while wearing thick socks further indicated and supported the importance of feedback from plantar tactile afferents in maintenance of balance, which is in accordance with previous studies reported that falling risks could be increased by the use of socks. ${ }^{4,6}$ This raises concern, particularly for those who are prone to falls, about the use of thick socks which could impair balance. The findings were in accordance with previous studies which reported reduced static balance after an intervention of plantar desensitization, achieved by exposing subjects' feet to ice for a few minutes ${ }^{30}$ or injecting anesthetic solution; ${ }^{31}$ and asking the subjects to stand on a soft foam surface. ${ }^{7}$ This study 
observed that socks alone could decrease postural stability, clinicians need to be aware of this when making clinical decisions.

Following the increase in all COP parameters as the effect of wearing thick socks, all COP parameters then significantly reduced while using the orthopaedic insoles with thick socks. In fact, no significant difference was found between the condition of barefoot and the condition of wearing both thick socks and insoles. This indicated that the negative balance effects of thick socks were dampened by the use of orthopaedic insoles. A previous study revealed that non-fallers tended to have smaller COP range (16.5\% and 14.2\% smaller in ML and AP directions, respectively) and mean velocity (15.0\% slower) compared with fallers ${ }^{24}$ during standing. Coincidently, this current study showed that the insoles reduced these parameters to similar percentages (reduction of approximate 15\%) in older adults. Additionally, it is interesting to note that the degree of improvement in postural stability after using orthopaedic insoles is similar to the condition of using higher-cost vibrating insoles. ${ }^{11}$ Future studies should investigate the potential effects of orthopaedic insole in balance improvement under more diverse medical and physical conditions. The effects of orthopaedic insoles on postural balance of people with impairments in plantar tactile sensation caused by aged degeneration, diabetic neuropathy, and rheumatoid arthritis ${ }^{3}$ under various characteristics of shoes soles and supporting surfaces can be investigated. Future studies should also look into the potential relationships among plantar tactile sensation, plantar forces, and balance; which are largely unexplored. This study did not involve a shoes condition concerning the possible confounding effect of footwear. Future studies could consider investigating the effect of socks and orthopaedic insoles on postural balance when subjects are wearing 
standardized shoes. In addition to orthopaedic insoles, some shoes with similar designs of heel cups and arch supports could also have the similar balance improving effect, which merits further investigation.

\section{Limitations of study}

This study recruited healthy older adults only. Future study could investigate the effect of orthopaedic insoles on balance in population with higher risk of falls. To eliminate the possible effects of shoes on postural balance, this study required all subjects to stand without any shoes on force plates when assessing the balance. Future studies should investigate the combined effects of orthoaedic insoles and different types of shoes on balance, since different physical properties of shoes were found to have affected balance. ${ }^{8}$

\section{Conclusions}

This study investigated the immediate effect of orthopaedic insoles on postural balance of older adults, whose balance was adversely affected by the use of thick socks. After using the thick socks, the subjects' static postural sway increased. This raises concern for people who are prone to falls about wearing thick socks. Upon using insoles, this study showed the subjects then exhibited significantly less static postural sway. Insoles with an arch support, a metatarsal pad, and a heel cup were traditionally used to treat foot pain and deformity. This study indicated their potential of an additional benefit of balance improvement. This sheds new light on the application of a low-cost and practical solution for improving balance, which appears not to have caught much attention.

Word count: 2677 words. 


\section{Acknowledgments}

This work was supported by The Hong Kong Polytechnic University Research

Studentship [grant number: RTNR], The Health and Medical Research Fund of Hong Kong SAR [grant number: HMRF11122231], and the Institute of Active Aging, The Hong Kong Polytechnic University.

\section{Declaration of Conflicting Interests}

The Authors declare that there is no conflict of interest.

\section{References}

1. Rubenstein LZ. Falls in older people: epidemiology, risk factors and strategies for prevention. Age and ageing. 2006; 35: ii37-ii41.

2. Kavounoudias A, Roll R and Roll J-P. The plantar sole is a 'dynamometric map’for human balance control. Neuroreport. 1998; 9: 3247-52.

3. Hennig EM and Sterzing T. Sensitivity mapping of the human foot: thresholds at 30 skin locations. Foot \& Ankle International. 2009; 30: 986-91.

4. Menant JC, Steele JR, Menz HB, Munro BJ and Lord SR. Optimizing footwear for older people at risk of falls. J Rehabil Res Dev. 2008; 45: 1167-81.

5. Kuklane K. Protection of feet in cold exposure. Industrial Health. 2009; 47: 24253. 
6. Tsai Y-J and Lin S-I. Older adults adopted more cautious gait patterns when walking in socks than barefoot. Gait \& posture. 2013; 37: 88-92.

7. Patel M, Fransson P, Lush D and Gomez S. The effect of foam surface properties on postural stability assessment while standing. Gait \& posture. 2008; 28: 649-56.

8. Hatton AL, Rome K, Dixon J, Martin DJ and McKeon PO. Footwear Interventions A Review of Their Sensorimotor and Mechanical Effects on Balance Performance and Gait in Older Adults. Journal of the American Podiatric Medical Association. 2013; 103: 516-33.

9. Maki BE, Sibley KM, Jaglal SB, et al. Reducing fall risk by improving balance control: Development, evaluation and knowledge-translation of new approaches. Journal of safety research. 2011; 42: 473-85.

10. Aruin AS and Kanekar N. Effect of a textured insole on balance and gait symmetry. Experimental brain research. 2013; 231: 201-8.

11. Priplata AA, Patritti BL, Niemi JB, et al. Noise-enhanced balance control in patients with diabetes and patients with stroke. Annals of neurology. 2006; 59: 4-12.

12. Conceição CSd, Gomes Neto M, Mendes SM, Sá KN and Baptista AF. Systematic review and meta-analysis of effects of foot orthoses on pain and disability in rheumatoid arthritis patients. Disability \& Rehabilitation. 2014: 1-5. 
13. Goff JD and Crawford R. Diagnosis and treatment of plantar fasciitis. American family physician. 2011; 84: 676.

14. Lee PY, Landorf KB, Bonanno DR and Menz HB. Comparison of the pressurerelieving properties of various types of forefoot pads in older people with forefoot pain. Journal of foot and ankle research. 2014; 7: 1.

15. Chen T-h, Chou L-W, Tsai M-W, Lo M-J and Kao M-J. Effectiveness of a heel cup with an arch support insole on the standing balance of the elderly. Clin Interv Aging. 2014; 9: 351-6.

16. de Morais Barbosa C, Bértolo MB, Neto JFM, Coimbra IB, Davitt M and de Paiva Magalhães E. The effect of foot orthoses on balance, foot pain and disability in elderly women with osteoporosis: a randomized clinical trial. Rheumatology. 2012: kes300.

17. Gross MT, Mercer VS and Lin F-C. Effects of foot orthoses on balance in older adults. The Journal of orthopaedic and sports physical therapy. 2012; 42: 649-57.

18. Takata Y, Matsuoka S, Okumura N, Iwamoto K, Takahashi M and Uchiyama E. Standing balance on the ground—the influence of flatfeet and insoles. J Phys Ther Sci. 2013; 25: 1519. 
19. Hamlyn C, Docherty CL and Klossner J. Orthotic intervention and postural stability in participants with functional ankle instability after an accommodation period. Journal of athletic training. 2012; 47: 130.

20. Bus SA, Ulbrecht JS and Cavanagh PR. Pressure relief and load redistribution by custom-made insoles in diabetic patients with neuropathy and foot deformity. Clinical Biomechanics. 2004; 19: 629-38.

21. Tyson S and Connell LA. How to measure balance in clinical practice. A systematic review of the psychometrics and clinical utility of measures of balance activity for neurological conditions. Clinical Rehabilitation. 2009.

22. Ma CZ-H, Wong DW-C, Lam WK, Wan AH-P and Lee WC-C. Balance improvement effects of biofeedback systems with state-of-the-art wearable sensors: a systematic review. Sensors. 2016; 16: 434.

23. Chaudhry H, Bukiet B, Ji Z and Findley T. Measurement of balance in computer posturography: Comparison of methods—A brief review. Journal of bodywork and movement therapies. 2011; 15: 82-91.

24. Melzer I, Kurz I and Oddsson LIE. A retrospective analysis of balance control parameters in elderly fallers and non-fallers. Clinical Biomechanics. 2010; 25: 984-8. 
25. Agrawal Y, Carey JP, Hoffman HJ, Sklare DA and Schubert MC. The modified Romberg Balance Test: normative data in US adults. Otology \& neurotology. 2011; 32: $1309-11$.

26. Ruhe A, Fejer R and Walker B. The test-retest reliability of centre of pressure measures in bipedal static task conditions-A systematic review of the literature. Gait \& posture. 2010; 32: 436-45.

27. Slater RA, Koren S, Ramot Y, Buchs A and Rapoport MJ. Interpreting the results of the Semmes-Weinstein monofilament test: accounting for false-positive answers in the international consensus on the diabetic foot protocol by a new model.

Diabetes/metabolism research and reviews. 2014; 30: 77-80.

28. Prieto TE, Myklebust J, Hoffmann R, Lovett E and Myklebust B. Measures of postural steadiness: differences between healthy young and elderly adults. IEEE Transactions on Biomedical Engineering. 1996; 43: 956-66.

29. Bell-Krotoski JA, Fess EE, Figarola JH and Hiltz D. Threshold detection and Semmes-Weinstein monofilaments. Journal of Hand Therapy. 1995; 8: 155-62.

30. Manor B, Wolenski P, Guevaro A and Li L. Differential effects of plantar desensitization on locomotion dynamics. Journal of Electromyography and Kinesiology. 2009; 19: e320-e8. 
31. Höhne A, Ali S, Stark C and Brüggemann G-P. Reduced plantar cutaneous sensation modifies gait dynamics, lower-limb kinematics and muscle activity during walking. Eur J Appl Physiol. 2012; 112: 3829-38. 
Table 1: Comparison of monofilament score with and without using socks among older adults $(\mathbf{n}=14)$.

\begin{tabular}{lccc}
\hline & Monofilament score & \\
\hline Testing site & Wean \pm SD) & \\
\hline Hallux & $3.0 \pm 0.0$ & $1.1 \pm 0.3$ & $<0.001^{*}$ \\
\hline 1st metatarsal head & $2.9 \pm 0.2$ & $1.1 \pm 0.2$ & $<0.001^{*}$ \\
\hline 5th metatarsal head & $2.6 \pm 0.6$ & $1.0 \pm 0.0$ & $<0.001^{*}$ \\
\hline Average & $2.8 \pm 0.3$ & $1.1 \pm 0.1$ & $<0.001^{*}$ \\
\hline
\end{tabular}

${ }^{1}$ Higher monofilament score indicates better sensation.

*: Significant difference was found. 
Table 2: Comparison of COP parameters in 3 experimental conditions in older adults (n=14).

\begin{tabular}{|c|c|c|c|c|c|c|c|c|c|}
\hline \multirow{2}{*}{$\begin{array}{c}\text { Measurements of } \\
\text { COP } \\
(\text { mean } \pm \text { SD) }\end{array}$} & \multicolumn{3}{|c|}{ Three testing conditions } & \multicolumn{2}{|c|}{ Difference } & \multicolumn{4}{|c|}{ P-value } \\
\hline & $\begin{array}{l}\text { No socks or } \\
\text { insoles } \\
\text { (Condition 1) }\end{array}$ & $\begin{array}{c}\text { With socks, no } \\
\text { insoles } \\
\text { (Condition 2) } \\
\end{array}$ & $\begin{array}{c}\text { With socks and } \\
\text { insoles } \\
\text { (Condition 3) }\end{array}$ & $\begin{array}{c}\text { Condition } 2 \\
\text { minus } \\
\text { Condition } 1 \\
\end{array}$ & $\begin{array}{c}\text { Condition } 3 \\
\text { minus } \\
\text { Condition } 2 \\
\end{array}$ & $\begin{array}{l}\text { One-way repeated } \\
\text { measures ANOVA }\end{array}$ & $\begin{array}{c}\text { Condition } 2 \text { vs } \\
\text { Condition } 1 \\
\text { (post hoc) }\end{array}$ & $\begin{array}{l}\text { Condition } 3 \text { vs } \\
\text { Condition } 2 \\
\text { (post hoc) } \\
\end{array}$ & $\begin{array}{c}\text { Condition } 3 \text { vs } \\
\text { Condition } 1 \\
\text { (post hoc) } \\
\end{array}$ \\
\hline $\begin{array}{c}\begin{array}{c}\text { Mean Distance } \\
(\mathrm{mm})\end{array} \\
\end{array}$ & $6.21 \pm 0.98$ & $8.27 \pm 1.59$ & $7.02 \pm 1.65$ & $+33.2 \%$ & $-15.0 \%$ & $<0.001^{*}$ & $<0.001^{*}$ & $<0.001^{*}$ & 0.120 \\
\hline $\begin{array}{l}\text { Root Mean Square } \\
\text { Distance }(\mathrm{mm})\end{array}$ & $7.11 \pm 1.09$ & $9.54 \pm 1.76$ & $8.02 \pm 1.87$ & $+34.2 \%$ & $-16.0 \%$ & $<0.001^{*}$ & $<0.001^{*}$ & $<0.001^{*}$ & 0.124 \\
\hline $\begin{array}{c}\text { Mean Velocity } \\
(\mathrm{mm} / \mathrm{s})\end{array}$ & $0.07 \pm 0.01$ & $0.09 \pm 0.02$ & $0.08 \pm 0.02$ & $+33.2 \%$ & $-15.0 \%$ & $<0.001^{*}$ & $<0.001^{*}$ & $<0.001^{*}$ & 0.120 \\
\hline Sway Area $\left(\mathrm{mm}^{2}\right)$ & $2.27 .71 \pm 466.65$ & $2569.98 \pm 614.71$ & $2209.13 \pm 580.26$ & $+26.7 \%$ & $-14.0 \%$ & $<0.001^{*}$ & $<0.001^{*}$ & $0.002 *$ & 0.318 \\
\hline $\begin{array}{c}95 \% \text { Confidence } \\
\text { Circle Area }\left(\mathrm{mm}^{2}\right)\end{array}$ & $460.02 \pm 132.39$ & $847.81 \pm 305.92$ & $596.92 \pm 305.09$ & $+84.3 \%$ & $-29.6 \%$ & $<0.001^{*}$ & $<0.001^{*}$ & $<0.001^{*}$ & 0.120 \\
\hline $\begin{array}{l}\text { 95\% Confidence } \\
\text { Ellipse Area }\left(\mathrm{mm}^{2}\right)\end{array}$ & $421.44 \pm 147.36$ & $847.95 \pm 338.92$ & $609.91 \pm 313.82$ & $+101.2 \%$ & $-28.1 \%$ & $<0.001^{*}$ & $0.001 *$ & $<0.001^{*}$ & 0.139 \\
\hline $\begin{array}{l}\text { Anterior-Posterior } \\
\text { Range (mm) }\end{array}$ & $37.34 \pm 8.63$ & $45.67 \pm 7.83$ & $39.68 \pm 8.38$ & $+22.3 \%$ & $-13.1 \%$ & $0.002 *$ & $0.002 *$ & $0.004 *$ & 0.980 \\
\hline $\begin{array}{c}\text { Medial-Lateral } \\
\text { Range }(\mathrm{mm})\end{array}$ & $30.67 \pm 9.09$ & $51.75 \pm 9.07$ & $38.47 \pm 7.64$ & $+68.7 \%$ & $-25.7 \%$ & $<0.001 *$ & $<0.001^{*}$ & $<0.001 *$ & 0.098 \\
\hline
\end{tabular}

*: Significant difference was found. 


\section{Figure Captions}

Fig 1: Design of the orthopaedic insole.

Fig 2: The COP trajectory in three different conditions in one elderly subject.

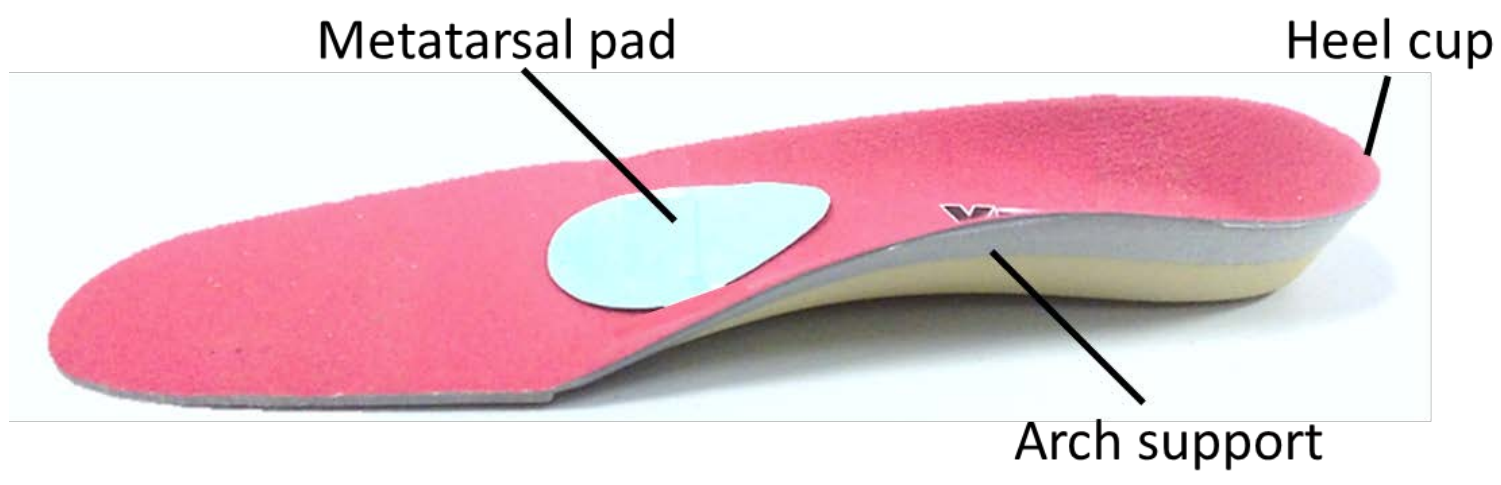

Fig 1: Design of the orthopaedic insole 
A. COP trajectory-no socks or insoles

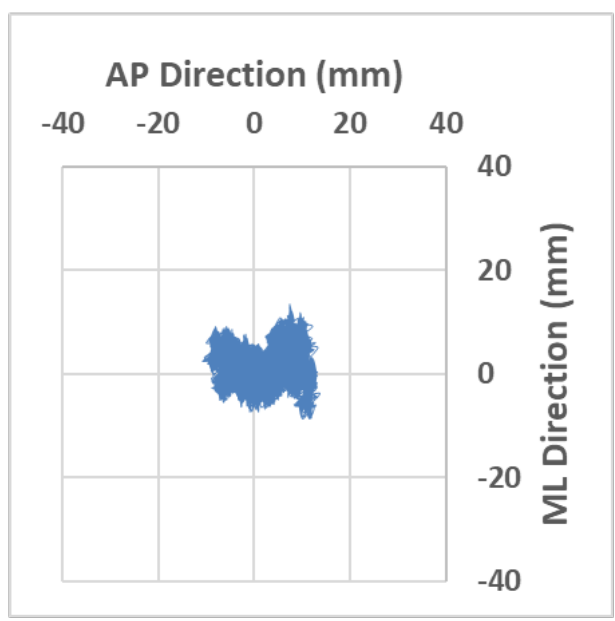

B. COP trajectory-with socks, no insoles

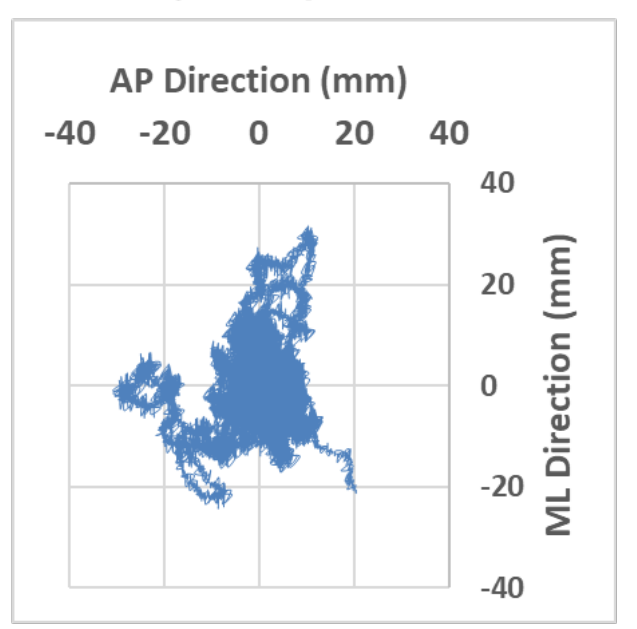

C. COP trajectory-with socks and insoles

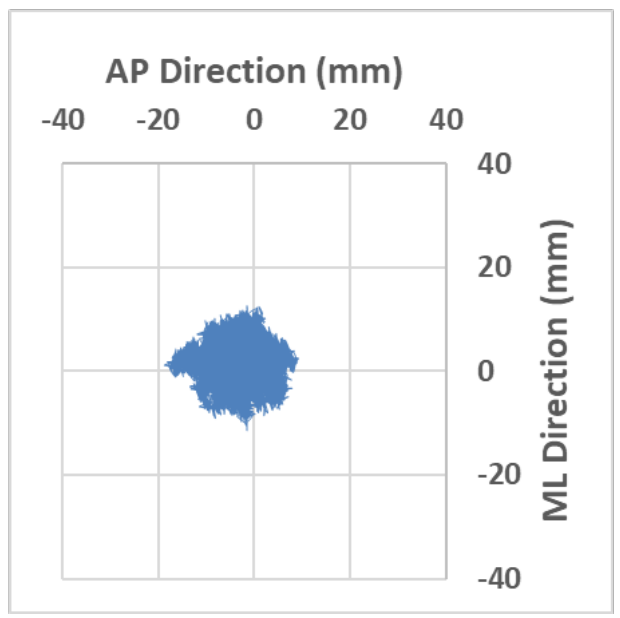

Fig 2: The COP trajectory in three different conditions in one elderly subject. 